\title{
Study of Light Trapping Scheme to Improve Performance of Silicon Solar Cells
}

\author{
R. Sircar ${ }^{1, *}$, J. Trivedi ${ }^{1}$, S. Gupta ${ }^{1}$, B. Tripathi ${ }^{2}$, D.P. Srivastava ${ }^{1}$ \\ 1 Photovoltaics Research Laboratory, Department of Physics, Feroze Gandhi College Raebareli - 229001, India \\ ${ }^{2}$ School of Solar Energy, Pandit Deendayal Petroleum University, Raisan, Gandhinagar - 382007, India
}

(Received 26 December 2015; published online 15 March 2016)

\begin{abstract}
The efficiency of solar cells can be increased by applying multidisciplinary approaches that encompass many branches of physics and engineering. One of the important branches of physics namely optics can be used for designing an scheme of light trapping for solar cells to reduce the reflection from the front surface and increasing the optical path length of light within the solar cell. In this paper, the potential of light trapping scheme has been studied and calculations have been done using PC1D base model with a solar cell having texturing on front and rear surface. A maximum output short-circuit current of 5.88 Amperes is obtained with front facet angle 700 and rear facet angle 600 for an area of $125 \mathrm{~mm} \times 125 \mathrm{~mm}$.
\end{abstract}

Keywords: Silicon solar cell, Light trapping, PC1D, Optical path length.

DOI:

PACS numbers: 71.23.An, 71.23.Cq

\section{INTRODUCTION}

In recent years, the development of clean and renewable energy sources has transcended from a subject of importance to researchers and environmentalists to one of the major subject of national interest that concerns economic progress, the welfare of working families, national energy security and saving the environment [1]. Due these requirements, the demand for improving the efficiency of light to electricity conversion has been continuously increasing. Due to the current trend towards the development of thinner silicon wafer-based solar cells, achieving higher efficiencies become even more challenging. A promising route to enhance the efficiency is to increase the absorption probability for the solar photons impinging on the photovoltaic device. However, the reduction in active photovoltaic material compromises the cell efficiency and consequently the need for optimized optical design arises [2, 3]. Using a better light trapping scheme, a greater degree of light absorption can be achieved. The more solar photons are absorbed by the active layer(s) of the solar cell, the more electron-hole pairs are generated which contribute to the electrical current and voltage output of the device.

Various light trapping approaches hold the capacity to reduce the thickness of the active solar cell material while still maintaining, in theory, similar absorption characteristics as that of thicker cells. A variety of approaches have been developed to minimize the reflection losses through modifying surface topography $[4,5]$ wherein the losses are reduced by surface texturing using anisotropic etching generally done in aqueous alkaline solution. However, this process is limited to single crystalline silicon (c-Si) only [6] and not very effective in multi-crystalline silicon (mc-Si) due to different orientations of grains. Porous silicon, formed by electrochemical etching, is another such approach to reduce reflection loss in the range of $5-8 \%$ [7]. This means that with the addition of an appropriate light trapping scheme the efficiencies of standard commercial cells can be achieved with much less material.
Therefore, photon management becomes essential. In this article, a study related to the textured front and back surface is done and the textured facet angle is optimized for improved absorption.

\section{POTENTIAL OF LIGHT TRAPPING}

Light trapping in solar cells mainly falls into two categories, the first being the reduction of front surface reflection and the second involves increasing the optical path length of light within the cell. In our study, we combine planar antireflective (AR) coatings with nano structured diffractive gratings to enhance the light trapping capabilities of the solar cells and hence increase the short circuit current characteristics of the device.

However when light wave encounters a medium with a different refractive index, nature dictates that the light will get reflected, transmitted or absorbed by the material medium. These three phenomena are in essence the fundamental principles that govern the science of light trapping. Hence, it is worthwhile to take a closer look at how the science of light trapping is employed to a silicon solar cell and observe the benefits associated with the addition of each optical structure. We begin with a light wave that propagates through free space and is normally incident on the surface of bare silicon (c-Si), i.e., silicon with no antireflective coating, as shown in Fig. 1. The first thing we observe is that a significant portion of the light is lost to reflection. This case can be viewed as the non-light trapping case; where only $40 \%$ gets absorbed, we can consider this as a basis for the effects of light trapping for purposes of illustrating the effects of adding the various optical components.

In the continuation of our discussion on reducing optical losses from a bare silicon slab, we introduce an anti-reflective coating (AR coating) as shown in Fig 2. An AR coating is nothing more than layers of mainly dielectric material that are applied to a surface to reduce the reflectance. The concept of reducing the surface reflectivity by introducing intermediate transparent layers has been known since the early 1800's [8].

*ratnasircar@gmail.com 
The absorbed light improves to $57 \%$, which is $17 \%$ more than that was absorbed for the bare silicon case. Again, as it was anticipated, there is now more light to transmit, and hence, the transmission increases by $10 \%$ to a value of $35 \%$, i.e., when compared to the bare silicon case. Usually, transparent quarter wavelength layers of silicon oxide $\left(\mathrm{SiO}_{x}\right)$, silicon nitride $\left(\mathrm{Si}_{x} \mathrm{~N}_{y}\right)$ or titanium oxide $\left(\mathrm{TiO}_{x}\right)$ with intermediate or gradient refractive indices are used as antireflection coating (ARC) [10]. These coatings have resonant structures and work effectively only in a limited spectral range. So, the next logical step in this train of thought is to minimize the transmission losses.

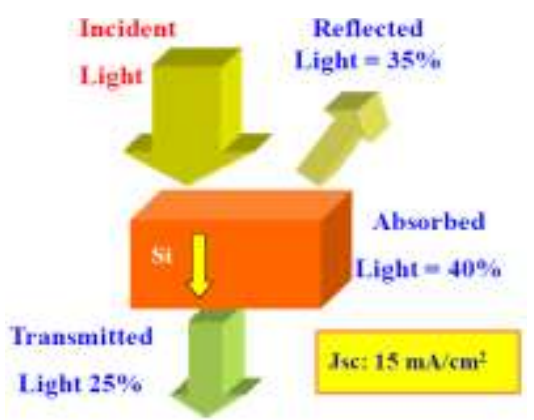

Fig. 1 - Schematic of a plane light wave incident on the surface of bare silicon [9]

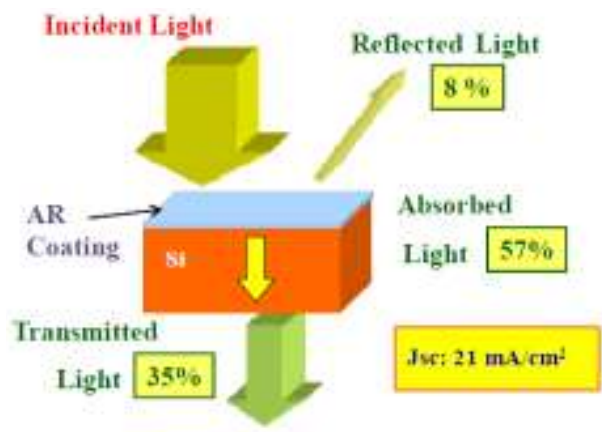

Fig. 2 - Schematic of a plane light wave incident on the surface of silicon with an added AR coating, only $8 \%$ of the incident light is now reflected, $57 \%$ is absorbed [9]

With the addition of an idealized back reflector, no light is transmitted and the light that would otherwise be lost gets reflected back into the active region where it can be reabsorbed as it passes through, as shown in Fig. 3, this time $33 \%$ of the light is reflected due to light that is reflected from the back surface. The optical path length of light is doubled in this case and the absorption improves to $67 \%$. There is no transmitted light. Now the total absorption increased by $10 \%$ as the path length doubles.

Hence, with the increased path length, we are essentially increasing the effective thickness of the active region, and thus allowing for more photons, with longer absorption depths, to be absorbed. But in this case reflected light is also increased sufficiently. The addition of the back reflector still does not do much for the light at the silicon band edge. We observe only minor increments of light absorption at those wavelengths and hence, this situation necessitates the addition of a more complex geometry. To this end, we incorporate a diffraction grating, to the back reflector.

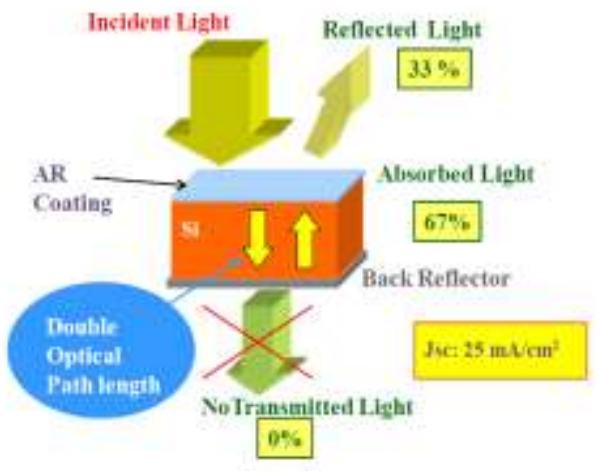

Fig. 3 - Schematic of a plane light wave incident on the surface of silicon with an added AR coating and back reflector [9]

The main idea, behind integrating a diffraction grating is to not only increase the path length of diffracted waves, but also to ensure that the waves remain in the silicon structure by means of total internal reflection (TIR). Total internal reflection occurs when a light wave travelling in a high index material, is obliquely incident on an interface between the high index and another lower index material. If the light wave is incident at or beyond a critical angle, it gets totally reflected back into the higher index medium. This TIR phenomenon follows from Snell's law of refraction, in the case where the angle of refraction is 90 degrees; since the argument of the sine function cannot exceed unity. It is worthy to note that this condition only holds for materials with identical permeabilities, which is true for most dielectrics [11]. The equation for the critical angle is:

$$
\theta_{c}=\sin ^{-1}\left(\frac{n_{2}}{n_{1}}\right)
$$

where, $n_{1}>n_{2}$

Now modify the diffraction grating equation, by incorporating the critical angle, and hence determine the period of the grating:

$$
d=\frac{m \lambda_{0}}{n_{s} \sin \theta_{c}}
$$

where, $\lambda_{0}$ is the wavelength of light propagating through free space, and ns is the refractive index of c-Si.

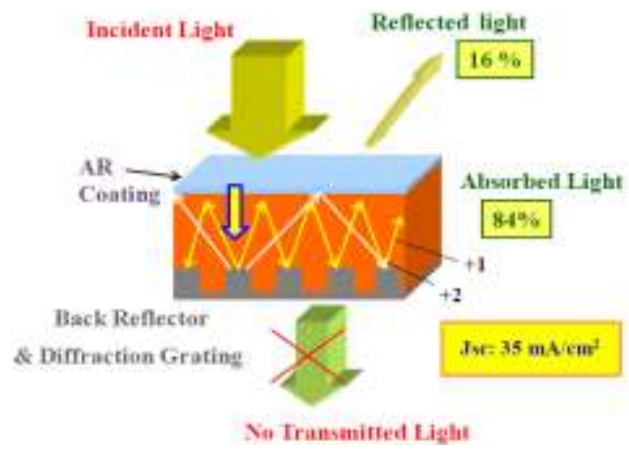

Fig. 4 - Schematic of a plane light wave incident on the surface of silicon with an added AR coating, back reflector and diffraction grating [9]

In the case of the structure with the added diffrac- 
tion grating as shown in Fig. 4, the absorption increases by a further $17 \%$ from the structure with a back reflector and AR coating only. It is worthy to note that the total absorption is more than double that of a structure with no added light trapping. There is also increased reflectance, due to the out coupling of zero order diffracted or specularly reflected light. Further enhancements in absorption can be achieved by varying the geometry of the grating.

\section{FRONT AND REAR SURFACE TEXTURING}

A common way of increasing conversion efficiency in silicon photovoltaic cell is to reduce reflection and enhance light confinement by texturing front/back surface. We have already done simulation work in our previous two papers $[12,13]$ and found the results for nano-texturing in front surface only. The highest values of electrical parameters were obtained for low texture depth (1-2 nm) and for facet angle values $86^{\circ}-87^{\circ}$. The efficiency could be increased nearby $5 \%$ of the efficiency value for base model without any texture.

In this paper we will discuss the effect of nanotexturing in both front and rear surface of thin film solar cell on their electrical output parameters. The schematic diagram of our base model is shown in fig. and device parameters are given in table as:

Table 1 - Device Parameters of Base Model

\begin{tabular}{|l|l|}
\hline Device Parameters & Values \\
\hline Area & $156.25 \mathrm{~cm}^{2}$ \\
\hline Thickness & $180 \mu \mathrm{m}$ \\
\hline Emitter contact & $1 \times 10^{-6} \Omega$ \\
\hline Base contact & $1.5 \times 10^{-2} \Omega$ \\
\hline Base doping $p$-type & $5 \times 10^{16} \mathrm{~cm}^{-3}$ \\
\hline Emitter doping $n$-type & $2.58 \times 10^{20} \mathrm{~cm}^{-3}$ \\
\hline Bulk recombination & $10 \mu \mathrm{s}$ \\
\hline Surface recombination & $S_{n}=1 \times 10^{4} \mathrm{~cm} / \mathrm{s}$, \\
velocity light source & $S_{p}=1 \times 10^{4} \mathrm{~cm} / \mathrm{s}$ \\
\hline Primary $\mathrm{W} \mathrm{cm-2}$, \\
Intensity Spectrum & $\mathrm{AM} 1.5$ \\
\hline
\end{tabular}

\section{SIMULATION RESULT AND DISCUSSION}

In this paper dependence of short circuit currents over front or rear facet angles are studied on $2 \mathrm{~nm}$ texture depth. In continuation with our previous work simulation has been done on rear surface as well as on front surface of solar cell. We plot graph in short circuit current with respect of rear facet angle at different front facet angles (Table 2 and Fig. 6).

Table 2-Short Circuit Current with different facet angles of front and rear surface

\begin{tabular}{|c|c|c|}
\hline \multirow{2}{*}{$\begin{array}{c}\text { Facet angle of } \\
\text { rear surface }\end{array}$} & \multicolumn{2}{|c|}{ ISC (Amperes) } \\
\cline { 2 - 3 } & $\begin{array}{l}\text { Front Facet } \\
\text { angle 70 }\end{array}$ & $\begin{array}{l}\text { Front Facet } \\
\text { angle 80 }\end{array}$ \\
\hline 10 & 5.788 & 5.778 \\
\hline 20 & 5.796 & 5.786 \\
\hline 30 & 5.808 & 5.798 \\
\hline 40 & 5.831 & 5.819 \\
\hline 50 & 5.861 & 5.837 \\
\hline 60 & 5.88 & 5.856 \\
\hline
\end{tabular}

\begin{tabular}{|l|l|l|}
\hline 70 & 5.875 & 5.851 \\
\hline 80 & 5.859 & 5.835 \\
\hline 82 & 5.846 & 5.822 \\
\hline 85 & 5.803 & 5.779 \\
\hline 89 & 5.718 & 5.694 \\
\hline
\end{tabular}

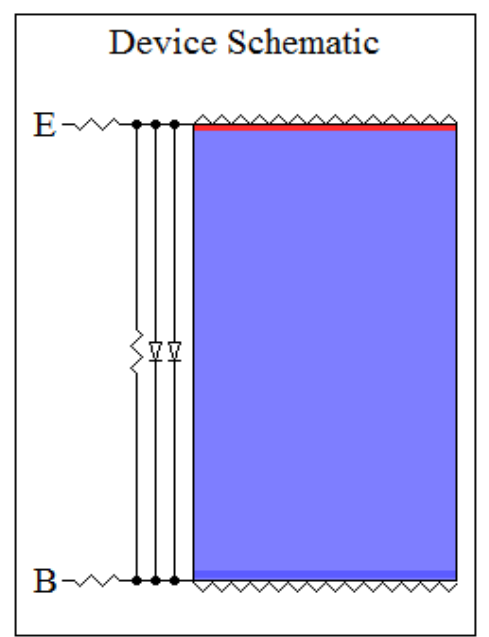

Fig. 5 - Schematic diagram of the device structure with front and rear texture, which is used as base model

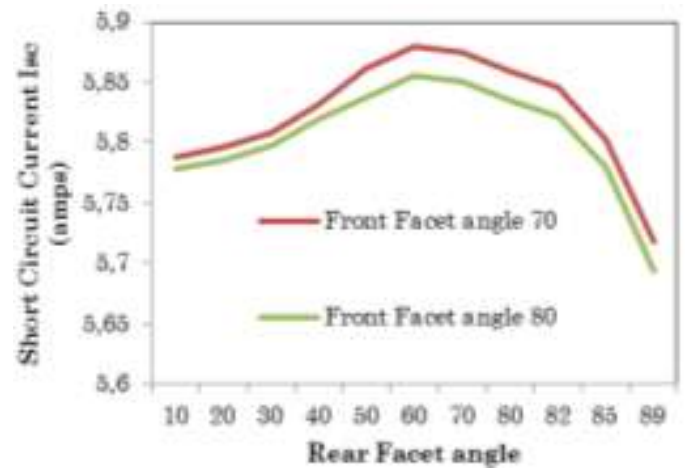

Fig. 6 - Plot showing variation of Short circuit current with rear facet angle for fixed front facet angle of $70^{\circ}$ and $80^{\circ}$

It is observe from the plot in Figure 6 that the maximum short circuit current is obtained at front facet angle 700 and rear facet angle 600. On the other hand if we consider only front surface texturing maximum Isc occur at front facet angle 860. The output parameters of chosen base model without texturing and with front and rear surface texturing are given in Table 3.

Table 3 - Comparison of output parameters without texturing and with front and rear surface texturing

\begin{tabular}{|c|l|l|r|}
\hline S. No. & $\begin{array}{l}\text { Output Parame- } \\
\text { ters }\end{array}$ & $\begin{array}{l}\text { Values of Base } \\
\text { Model No texturing }\end{array}$ & \multicolumn{2}{|c|}{$\begin{array}{l}\text { With } \\
\text { and Rear } \\
\text { face Textur }\end{array}$} \\
\hline 1 & $\begin{array}{l}\text { Short-circuit cur- } \\
\text { rent }\end{array}$ & $5.78 \mathrm{Amp}$ & $5.88 \mathrm{Am}$ \\
\hline 2 & $\begin{array}{l}\text { Maximum base } \\
\text { power }\end{array}$ & 2.575 Watt & $2.587 \mathrm{~W}$ \\
\hline 3 & $\begin{array}{l}\text { Open-circuit volt- } \\
\text { age }\end{array}$ & 0.6109 Volts & 0.6110 \\
\hline 4 & Fill factor (\%) & $72.9 \%$ & $72.1 \%$ \\
\hline 5 & Efficiency (\%) & $16.47 \%$ & $16.58 \%$ \\
\hline
\end{tabular}




\section{CONCLUSIONS}

In this paper the fundamental concepts of light trapping are discussed and their applications to c-Si solar cell devices are studied. The effect of nanotexturing in front as well as rear surface of c-Si solar cells on short circuit current is reported using PC1D simulation software. The results show that a maximum short-circuit current $\left(I_{S C}\right)$ of 5.88 Amperes is obtained

\section{REFERENCE}

1. B. Obama, Transcript: Obama's inaugural address (2009).

2. R. Brendel, A. Goetzberger, Thin-Film Crystalline Silicon Solar Cells: Physics and Technology (Weinheim: WileyVCH: 2003).

3. A. Luque, S. Hegedus, Handbook of Photovoltaic Science and Engineering (John Wiley \& Sons: 2003).

4. J. Zhao, A. Wang, M.A. Green, F. Ferrazza, Appl. Phys. Lett. 73, 1991 (1998).

5. P.K. Singh, R. Kumar, M. Lal, S.N. Singh, B.K. Das, Sol. Energ. Mater. Sol. C. 70, 103 (2001).

6. M.A. Green, Silicon Solar Cells: Advanced Principles and Practice (Bridge: Sydney: 1995).

7. P. Menna, G.D. Francia, V.L. Ferrara, Sol. Energ. Mater. Sol. C. 37, 13 (1995).

8. L. Rayleigh, Proc. London Math. Soc. 11, 51 (1879). with front facet angle of $70^{\circ}$ and rear facet angle of $60^{\circ}$ for an area of $125 \mathrm{~mm} \times 125 \mathrm{~mm}$. Improved efficiency of $16.58 \%$ is reported with front surface texturing, where ISC is obtained as 5.853 Amperes for a facet angle of $86^{\circ}$. This study reveals that the nano-texture improves the electrical performance by increasing absorption of incident radiation to efficient light trapping.

9. James Gichuhi Mutitu, Light Trapping Designs for Thin Silicon Solar Cells (Thesis in University of Delaware: 2010).

10. B.S. Richards, Prog. Photovolt.: Res. Appl. 12, 253 (2004).

11. C. Balanis, Adv. Eng. Electromagn (Wiley: 1989).

12. Shraddha Gupta, Dibya Prakash Srivastava, Brijesh Tripathi, Ratna Sircar, Jyotsana Trivedi, International Conference on Transport and Optical Properties of Nanomaterials (ICTOPON) (January 5-8, Allahabad, India, 2009).

13. Ratna Sircar, Brijesh Tripathi, Divya Prakash Srivastava, Shraddha Gupta, JyotsanaTrivedi, Role of Nano-Texturing to Improve Efficiency of Mc-Silicon Solar Cell (Seminar held at Prasad Institute of Management \& Technology) (Lucknow: 2012). 